\title{
CORRESPONDENCE Schizophrenia and neighbourhood deprivation
}

Translational Psychiatry (2016) 6, e979; doi:10.1038/tp.2016.244; published online 13 December 2016

Sariaslan et al. ${ }^{1}$ found that genetic risk for schizophrenia predicts neighbourhood deprivation, which they interpret as evidence against environmental causes for this association. However, their findings are consistent with a causal association between schizophrenia and later social deprivation.

The prevalence of schizophrenia is strongly socially patterned, being higher in more deprived sections of society. One possible explanation for this is that deteriorating mental state results in lower social position (social drift). Another is that social position is a contributing factor for mental illness (social causation). Sariaslan et al. ${ }^{1}$ used a polygenic risk score for schizophreni, and sibling and twin designs to capture genetic variation, to examine the hypothesis that patients with psychosis experience downward social mobility as a consequence of their symptoms. They found that both being a sibling of a schizophrenia proband and schizophrenia risk captured by the genetic risk score predicted neighbourhood deprivation, and concluded that this provides evidence against environmental causes for this association, and in support of genetic influences. They argue for a genetic selection interpretation, whereby genetic liability for schizophrenia predicts subsequent social position, reflected in neighbourhood deprivation.

These findings are also consistent with a causal association between behaviours seen in those at high risk of schizophrenia and subsequent social position. Genetic risk score analysis is akin to a Mendelian randomization design, ${ }^{2}$ whereby genetic variants are used as a proxy for an exposure of interest, to assess the unconfounded association between exposure and outcome. A genetic variant or risk score robustly associated with an exposure of interest could serve as an unconfounded proxy for behaviours of the kind seen among those at high risk of schizophrenia, as we inherit our genetic information independently of environmental factors, and each gene is inherited largely independently of other genes.

Schizophrenia is a rare outcome (and if effects on social mobility are only seen among those who develop schizophrenia, this could not generate an effect of the magnitude seen), but it is plausible that a higher genetic risk score could predict subclinical psychoticlike symptoms in the healthy population. Although evidence is limited, genetic risk for schizophrenia predicts dropout from the Avon Longitudinal Study of Parents and Children, ${ }^{3}$ which could reflect, for example, a more chaotic lifestyle. These subclinical psychotic-like experiences could influence the risk of social drift, without necessarily reaching the threshold for a clinical diagnosis. Indeed, in the same study, polygenic risk for schizophrenia predicts childhood psychopathy. ${ }^{4}$ There is a positive association between risk score and psychotic experiences at the most liberal $P$-value threshold, while at the most stringent threshold this seems to reverse. This could be an example of selection bias, where those at the highest biological risk of schizophrenia are more likely to have left the study, ${ }^{3}$ so that the individuals with the highest biological risk who remain in the study may be unusually healthy. Psychotic-like experiences or unusual behaviour and experiences might therefore plausibly negatively impact upon social position and lead to downward drift. A recent twin study suggested that positive psychotic experiences are associated with stressful life events in adolescence, and that genetic influences explain most of this association. ${ }^{5}$ This suggests a possible mechanism whereby genetic risk for schizophrenia could impact on the occurrence of stressful life events, and in turn social drift.

The distinction between biological and mediated pleiotropy is critical here. ${ }^{6}$ Biological (or horizontal) pleiotropy refers to a genetic variant influencing multiple phenotypes separately, via distinct biological pathways (Figure 1a). Mediated (or vertical) pleiotropy refers to a genetic variant influencing multiple outcomes via one biological pathway (for example, genetic effects on smoking behaviour also influencing lung cancer outcomes, via smoking $)^{7}$ (Figure $1 \mathrm{~b}$ ). In the case of mediated pleiotropy, other influences on the upstream phenotype (for example, smoking) would influence the downstream outcome (for example, lung cancer). Genetic studies can therefore provide insights into the modifiable causes of health and social outcomes. ${ }^{8}$

Sariaslan et al. interpret their bivariate quantitative genetic twin models as indicating that genetic influences account for the association between schizophrenia and neighbourhood deprivation rather than environmental factors, and argue that this is evidence against an environmental cause of social drift associated with schizophrenia. However, these findings are consistent with a causal relationship between behaviours related to schizophrenia risk and neighbourhood deprivation. Where the same genetic variants appear to predict two outcomes, this could suggest shared genetic aetiology, as the authors conclude, but it could also reflect a mediated causal relationship. ${ }^{8}$

In our opinion, further research to determine whether genetic risk for schizophrenia influences social position via behaviours associated with that risk is warranted. The crucial point is that, if a

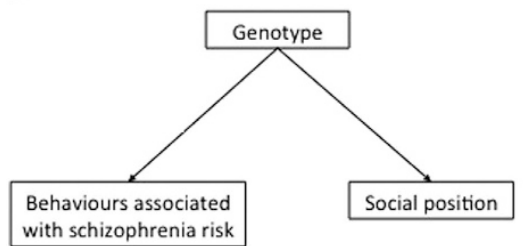

b

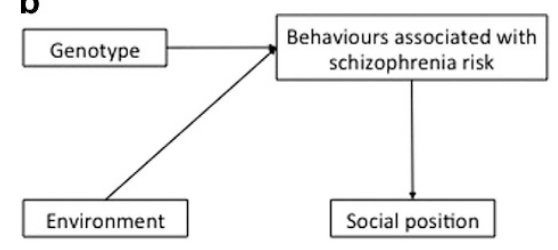

Figure 1. Directed acyclical graphs showing (a) shared genetic architecture (biological or horizontal pleiotropy), where genotype influences two separate phenotypes, and (b) mediated (or vertical) pleiotropy, where genotype impacts on one phenotype, which itself causally affects the second phenotype. In this instance, environmental factors can also impact on the first phenotype, meaning that there is the possibility for targeted interventions that can minimize the first phenotype (in this case behaviours associated with schizophrenia risk), which would also have a downstream effect on the second (social position). 
correct, our interpretation implies that it is behaviours present in those at high risk of schizophrenia that are causally related to social position. This could represent a modifiable target for intervention-since such behavioural patterns are influenced by factors other than genetics - and provide crucial insights to policy makers and clinicians. Once genetic results are interpreted through the lens of Mendelian randomization, they are no longer informative only about genetic influences, but also about modifiable influences. Since the genomewide association study used by Sariaslan and colleagues to generate a genetic risk score for schizophrenia was published, a larger study has identified 108 genetic variants at genomewide significance, explaining substantially more of the variation in schizophrenia risk. ${ }^{9}$ This could be utilized to explore how high genetic risk of schizophrenia impacts a non-clinical sample with higher power, and explore these relationships in more detail, to untangle causality.

\section{CONFLICT OF INTEREST}

This work was supported by the Medical Research Council Integrative Epidemiology Unit at the University of Bristol (MC_UU_12013/6). The authors declare no conflict of interest.

\author{
SH Gage ${ }^{1,2}$, G Davey Smith ${ }^{1}$ and MR Munafò̀ ${ }^{1,2}$ \\ ${ }^{1} \mathrm{MRC}$ Integrative Epidemiology Unit, University of Bristol, \\ Bristol, UK and \\ ${ }^{2}$ School of Experimental Psychology, University of Bristol, Bristol, UK \\ E-mail: Suzi.Gage@bristol.ac.uk
}

\section{REFERENCES}

1 Sariaslan A, Fazel S, D'Onofrio BM, Langstrom N, Larsson H, Bergen SE et al. Schizophrenia and subsequent neighborhood deprivation: revisiting the social drift hypothesis using population, twin and molecular genetic data. Transl Psychiatry 2016; 6: e796.

2 Davey Smith G. Mendelian randomization for strengthening causal inference in observational studies: application to gene $\mathrm{x}$ environment interactions. Perspect Psychol Sci 2010; 5: 527-545.

3 Martin J, Tilling K, Hubbard L, Stergiakouli E, Thapar A, Davey Smith G et al. Association of genetic risk for schizophrenia with nonparticipation over time in a population-based cohort study. Am J Epidemiol 2016; 183: 1149-1158.

4 Jones HJ, Stergiakouli E, Tansey KE, Hubbard L, Heron J, Cannon M et al. Phenotypic manifestation of genetic risk for schizophrenia during adolescence in the general population. JAMA Psychiatry 2016; 73: 221-228.

5 Shakoor S, Zavos HM, Haworth CM, McGuire P, Cardno AG, Freeman D et al. Association between stressful life events and psychotic experiences in adolescence: evidence for gene-environment correlations. Br J Psychiatry 2016; 208: 532-538.

6 Davey Smith G, Hemani G. Mendelian randomization: genetic anchors for causal inference in epidemiological studies. Hum Molec Genet 2014; 23: R89-R98.

7 Munafo MR, Timofeeva MN, Morris RW, Prieto-Merino D, Sattar N, Brennan P et al. Association between genetic variants on chromosome $15 q 25$ locus and objective measures of tobacco exposure. J Natl Cancer Inst 2012; 104: 740-748.

8 Gage SH, Davey Smith G, Ware JJ, Flint J, Munafo MR. G = E: What GWAS can tell us about the environment. PLoS Genet 2016; 12: e1005765.

9 Schizophrenia-Working-Group-of-the-Psychiatric-Genomics-Consortium. Biological insights from 108 schizophrenia-associated genetic loci. Nature 2014; 511: 421-427.

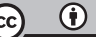

This work is licensed under a Creative Commons Attribution 4.0 International License. The images or other third party material in this article are included in the article's Creative Commons license, unless indicated otherwise in the credit line; if the material is not included under the Creative Commons license, users will need to obtain permission from the license holder to reproduce the material. To view a copy of this license, visit http://creativecommons.org/licenses/ by/4.0/

(c) The Author(s) 2016 